\title{
OP15
}

\section{DEFINITION OF A SPECTRAL MISMATCH INDEX FOR SPECTRAL POWER DISTRIBUTIONS}

\author{
Alejandro Ferrero et al. \\ DOI 10.25039/x46.2019.OP15 \\ from \\ CIE x046:2019 \\ Proceedings \\ of the \\ 29th CIE SESSION \\ Washington D.C., USA, June 14 - 22, 2019 \\ (DOI 10.25039/x46.2019)
}

The paper has been presented at the 29th CIE Session, Washington D.C., USA, June 14-22, 2019. It has not been peer-reviewed by CIE.

(c) CIE 2019

All rights reserved. Unless otherwise specified, no part of this publication may be reproduced or utilized in any form or by any means, electronic or mechanical, including photocopying and microfilm, without permission in writing from CIE Central Bureau at the address below. Any mention of organizations or products does not imply endorsement by the CIE.

This paper is made available open access for individual use. However, in all other cases all rights are reserved unless explicit permission is sought from and given by the CIE.

CIE Central Bureau

Babenbergerstrasse 9

A-1010 Vienna

Austria

Tel.: +4317143187

e-mail: ciecb@cie.co.at

www.cie.co.at 


\title{
DEFINITION OF A SPECTRAL MISMATCH INDEX FOR SPECTRAL POWER DISTRIBUTIONS
}

\author{
Ferrero, A. ${ }^{1}$, Kokka, A. ${ }^{2}$, Pulli, T. ${ }^{2}$, Poikonen, T. $^{3}$, Schneider, T. ${ }^{4}$, Stuker, F. ${ }^{5}$, Blattner, P. ${ }^{5}$, Pons, A. ${ }^{1}$, \\ Ikonen, E., ${ }^{2,3}$ \\ ${ }^{1}$ CSIC, Instituto de Óptica 'Daza de Valdés', Madrid, SPAIN, ${ }^{2}$ Metrology Research Institute, Aalto \\ University, Espoo, FINLAND, ${ }^{3}$ VTT Technical Research Centre of Finland Ltd, Espoo, FINLAND, \\ ${ }^{4}$ Instrument Systems GmbH, Munich, GERMANY, ${ }^{5}$ METAS, Bern-Wabern, SWITZERLAND
}

alejandro.ferrero@csic.es

DOI $10.25039 / \times 46.2019 .0 P 15$

\begin{abstract}
To investigate, select and publish an LED reference spectrum to complement the CIE Standard Illuminant $A$ in photometric calibrations, a new technical committee has been recently created by CIE. This technical committee requires defining a spectral mismatch index to quantify the match of spectral power distributions (SPDs) of real sources to the selected reference spectrum. A proposal for such an index is given here, assuming that the spectral mismatch index of the SPD of a real source is directly related to the spectral mismatch systematic error introduced when the photometric quantity is measured using a photometer which is calibrated to a light source with the reference SPD. Finally, the value of this index is discussed and interpreted in photometric terms.
\end{abstract}

Keywords: Photometry, LED sources, LED reference spectrum, photometric calibrations, spectral mismatch

\section{Introduction}

The technical committee TC 2-90 ("LED Reference spectrum for photometer calibration") has been recently created by $\mathrm{CIE}$ to investigate, select and publish a LED reference spectrum [Kokka 2018] to complement the CIE Standard Illuminant A in photometric calibrations. This task additionally requires defining a spectral mismatch index to quantify the match between spectral power distributions (SPDs) of real sources and the selected reference spectrum. The objective of this work is to propose such a spectral mismatch index, and to evaluate its performance using real and representative LED SPDs and relative spectral responsivities of photometers.

Since the reference SPD was proposed for photometric calibrations [Kokka 2018], the spectral mismatch index of the SPD of a real source should be directly related to the spectral mismatch systematic error introduced when the photometric quantity is measured by a photometer calibrated with respect to a light source with the reference SPD. This error is related to the spectral mismatch correction factor (SMCF).

According to ISO/CIE 19476 [ISO/CIE 2014], the expression of the SMCF is:

$$
F^{*}\left(S_{\mathrm{T}}\right)=\frac{\int_{360 \mathrm{~nm}}^{830 \mathrm{~nm}} S_{\mathrm{T}}(\lambda) \cdot V(\lambda) \mathrm{d} \lambda \int_{\lambda_{\min }}^{\lambda_{\max }} S_{\mathrm{R}}(\lambda) \cdot S_{\text {rel }}(\lambda) \mathrm{d} \lambda}{\int_{\lambda_{\min }}^{\lambda_{\max }} S_{\mathrm{T}}(\lambda) \cdot S_{\text {rel }}(\lambda) \mathrm{d} \lambda \int_{360 \mathrm{~nm}}^{830 \mathrm{~nm}} S_{\mathrm{R}}(\lambda) \cdot V(\lambda) \mathrm{d} \lambda}
$$

where $V(\lambda)$ is the luminous efficiency function of the human visual system, $s_{r e l}(\lambda)$ is the relative spectral responsivity of the photometer and $S_{T}(\lambda)$ and $S_{R}(\lambda)$ are test and reference SPDs, respectively. This expression equals 1 (no correction required) if $V(\lambda)$ and $s_{\text {rel }}(\lambda)$ or $S_{T}(\lambda)$ and $S_{R}(\lambda)$ have identical spectral distributions.

\section{Index definition}

Notice that the SMCF can be written as: 


$$
F^{*}\left(S_{\mathrm{T}}\right)=\frac{\frac{\int_{\lambda_{\min }}^{\lambda_{\max }} S_{\mathrm{R}}(\lambda) \cdot V(\lambda) \cdot \varepsilon(\lambda) \mathrm{d} \lambda}{\int_{360 \mathrm{~nm}}^{830 \mathrm{~nm}} S_{\mathrm{R}}(\lambda) \cdot V(\lambda) \mathrm{d} \lambda}}{\frac{\int_{\lambda_{\min }}^{\lambda_{\max }} S_{\mathrm{T}}(\lambda) \cdot V(\lambda) \cdot \varepsilon(\lambda) \mathrm{d} \lambda}{\int_{360 \mathrm{~nm}}^{830 \mathrm{~nm}} S_{\mathrm{T}}(\lambda) \cdot V(\lambda) \mathrm{d} \lambda}}
$$

where $\varepsilon(\lambda)$ is defined as:

$$
\varepsilon(\lambda)=\frac{s_{\text {rel }}(\lambda)}{V(\lambda)}
$$

which contains the information on variations in the relative spectral response with respect to $V(\lambda)$ of different photopic filters used in photometers.

According to this notation, the SMCF can be simply expressed as:

$$
F^{*}\left(S_{\mathrm{T}}\right)=\frac{E_{\mathrm{R}}[\varepsilon]}{E_{\mathrm{T}}[\varepsilon]}
$$

where $E_{x}[\varepsilon]$ is weighted arithmetic mean value of the variable $\varepsilon$ using as weighting function:

$$
p_{\mathrm{X}}(\lambda)=\frac{S_{\mathrm{X}}(\lambda) \cdot V(\lambda)}{\int_{360 \mathrm{~nm}}^{830 \mathrm{~nm}} S_{\mathrm{X}}(\lambda) \cdot V(\lambda) \mathrm{d} \lambda}
$$

Therefore, according to Eq. (4), SMCF can be regarded as the ratio between the weighted arithmetic mean of $\varepsilon(\lambda)$ when using the SPD of the reference source (R) and its weighted arithmetic mean when using the SPD of the source to be tested (T).

No correction is required $\left(F^{*}=1\right)$, regardless of the value of $\varepsilon$, when the functions $p_{R}(\lambda)$ and $p_{\mathrm{T}}(\lambda)$ coincide, because in that case $E_{\mathrm{R}}[\varepsilon]=E_{\mathrm{T}}[\varepsilon]$. On the other hand, the more different $p_{\mathrm{T}}(\lambda)$ is from $p_{R}(\lambda)$, the larger is usually the required correction $F^{*}$. Therefore, the dissimilarity between $p_{R}(\lambda)$ and $p_{T}(\lambda)$ was selected as basis for the spectral mismatch index.

To find out the optimal way to describe the differences between $p_{R}(\lambda)$ and $p_{T}(\lambda), 1$-norm and 2-norm distances of 1298 LED sources to the reference SPD were compared with the results obtained for the average values of $\left|1-F^{\star}\right|$ for the same source and reference SPD pairs over 77 different spectral responsivities Srel $(\lambda)$ [Kokka 2018] (giving in total around 100000 data points). The calculated values of $\left|1-F^{*}\right|$ were ranging from $1.5 \times 10^{-4}$ to $1.5 \times 10^{-2}$, thus covering two decades of correction factor values.

It was found that the 1-norm distance:

$$
\delta_{\mathrm{SM}}\left(p_{\mathrm{T}}\right)=\int_{360 \mathrm{~nm}}^{830 \mathrm{~nm}}\left|p_{\mathrm{T}}-p_{\mathrm{R}}\right| \mathrm{d} \lambda
$$

correlates slightly better with the average $\left|1-F^{*}\right|$ compared to the 2-norm metric:

$$
\delta_{\mathrm{SM} 2}\left(p_{\mathrm{T}}\right)=\int_{360 \mathrm{~nm}}^{830 \mathrm{~nm}}\left(p_{\mathrm{T}}-p_{\mathrm{R}}\right)^{2} \mathrm{~d} \lambda
$$

with linear correlation coefficients of 0.857 and 0.809 , respectively (see Figure 1). 

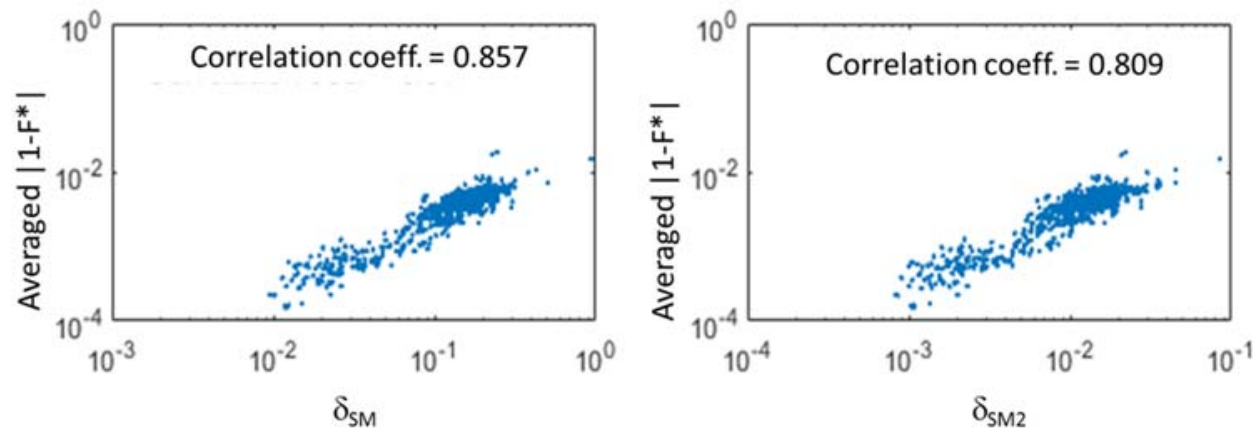

Figure 1 - Correlation of the average values of $\left|1-F^{\star}\right|$ with $\delta \mathrm{sm}$ and $\delta \mathrm{sm} 2$. Both axes are in logarithmic scale.

Therefore Eq. (6) is proposed as spectral mismatch index. It can be expressed more explicitly as:

$$
\delta_{\mathrm{SM}}\left(S_{\mathrm{T}}\right)=\int_{360 n m}^{830 n m}\left|\frac{S_{\mathrm{T}}(\lambda) \cdot V(\lambda)}{\int_{360 n m}^{830 n m} S_{\mathrm{T}}(\lambda) \cdot V(\lambda) \mathrm{d} \lambda}-\frac{S_{\mathrm{R}}(\lambda) \cdot V(\lambda)}{\int_{360 n m}^{830 n m} S_{\mathrm{R}}(\lambda) \cdot V(\lambda) \mathrm{d} \lambda}\right| \mathrm{d} \lambda
$$

The higher its value, the higher the expected spectral mismatch is when using a photometer with an unknown spectral responsivity.

\section{Practical considerations}

It is important to understand the meaning of a specific value of the SPD spectral mismatch index. The question to answer is:

If my light source has a SPD spectral mismatch index of $\delta \mathrm{SM}$, how much correction does the response of a photometer of quality $Q$ potentially require to match exactly its hypothetical response when calibrated with the reference SPD?

Based on the strong linear correlation, between the SPD spectral mismatch index $\delta_{\mathrm{SM}}$ and $\mid 1$ $F^{*} \mid$, also strong linear correlations of $\delta_{\mathrm{SM}}$ with statistical moments of $\left|1-F^{*}\right|$ (mean, standard deviation, percentiles) can be expected when $\delta_{\mathrm{SM}}$ and $\left|1-F^{*}\right|$ are evaluated for a large data sets of LED sources and of photometers on the market.

For the SPDs and spectral responsivities from [Kokka 2018], the $95^{\text {th }}$ and $50^{\text {th }}$ percentiles of $\mid 1$ - $F^{*} \mid\left(P_{50}\right.$ and $P_{95}$, respectively) are used to estimate the factors $f_{50}$ and $f_{95}$ for which:

$$
\begin{aligned}
& P_{50}\left(\left|1-F^{*}\right|\right)=f_{50} \delta_{\mathrm{SM}} \\
& P_{95}\left(\left|1-F^{*}\right|\right)=f_{95} \delta_{\mathrm{SM}}
\end{aligned}
$$

To assess the dependence on the quality $Q$ of the photometer, the spectral responsivities of the photometers were separated in different classes according to their photometric performance, following CIE TC2-69 [CIE 2019]:

- Class $4^{*}$ : Meters with highest possible accuracy (laboratory measurements): $f_{1}<$ $1,5 \%$

- Class $3^{*}$ : Meters with high levels of accuracy: $1,5 \%<f_{1} \leq 3 \%$

- Class $2^{*}$ : Meters with medium levels of accuracy: $3 \%<f_{1} \leq 6 \%$

- Class $1^{*}$ : Meters with low levels of accuracy (rough measurements): $6 \%<f_{1} \leq 9 \%$ 
with $f_{1}$ defined as in [ISO/CIE 2014]:

$$
f_{1}{ }^{\prime}=\frac{\int_{380 \mathrm{~nm}}^{780 \mathrm{~nm}}\left|S_{\text {rel }}^{*}(\lambda)-V(\lambda)\right| \mathrm{d} \lambda}{\int_{380 \mathrm{~nm}}^{780 \mathrm{~nm}} V(\lambda) \mathrm{d} \lambda}
$$

where $s_{\text {rel }}^{*}(\lambda)$ is the normalized spectral responsivity function:

$$
\begin{aligned}
& S_{\text {rel }}^{*}(\lambda)=S_{\text {rel }}(\lambda) \cdot \frac{\int_{780 \mathrm{~nm}}^{780 \mathrm{~nm}} S_{\mathrm{A}}(\lambda) V(\lambda) \mathrm{d} \lambda}{780 \mathrm{~mm}} \\
& \int_{380 \mathrm{~nm}} S_{\mathrm{A}}(\lambda) S_{\text {rel }}(\lambda) \mathrm{d} \lambda
\end{aligned}
$$

being $S_{A}$ the spectral power distribution of the CIE Standard Illuminant $A$.

Notice that Eqs. (11)-(12) can be rearranged as:

$$
{f^{\prime}}_{1}=\int_{380 \mathrm{~nm}}^{780 \mathrm{~nm}}\left|\frac{S_{\text {rel }}(\lambda)}{\int_{380 \mathrm{~nm}}^{780 \mathrm{~S}} \hat{S}_{\mathrm{A}}(\lambda) \cdot s_{\text {rel }}(\lambda) \mathrm{d} \lambda}-\frac{V(\lambda)}{\int_{380 \mathrm{~nm}}^{780 \mathrm{~S}} \hat{S}_{\mathrm{A}}(\lambda) \cdot V(\lambda) \mathrm{d} \lambda}\right| \mathrm{d} \lambda
$$

to show that this index for photometers is very similarly defined to the proposed one for light sources in Eq. (8). $\hat{S}_{\mathrm{A}}$ is $S_{\mathrm{A}}$ normalized to its $V(\lambda)$-weighted integration $\frac{\int_{380 \mathrm{~nm}}^{780 \mathrm{~nm}} S_{\mathrm{A}}(\lambda) \cdot V(\lambda) \mathrm{d} \lambda}{\int_{380 \mathrm{~nm}}^{70} V(\lambda) \mathrm{d} \lambda}$.

$f_{50}$ and $f_{95}$ were calculated for each class, using the LED illuminant BL40 from [Kokka 2018], a white LED spectrum with a CCT of about $4100 \mathrm{~K}$ which was recently proposed as LED reference spectrum for photometric calibration by CIE TC2-90. 10 of the available photometers are $4^{*}, 32$ are $3^{*}, 23$ are $2^{*}$ and 12 are $1^{*}$. Some examples of distributions of $\mid 1$ $F^{*} \mid$ are given from Figure 2 to Figure 5 . They correspond to the LEDs with SPDs shown in Figure 6, where the proposed reference LED SPD is also shown.
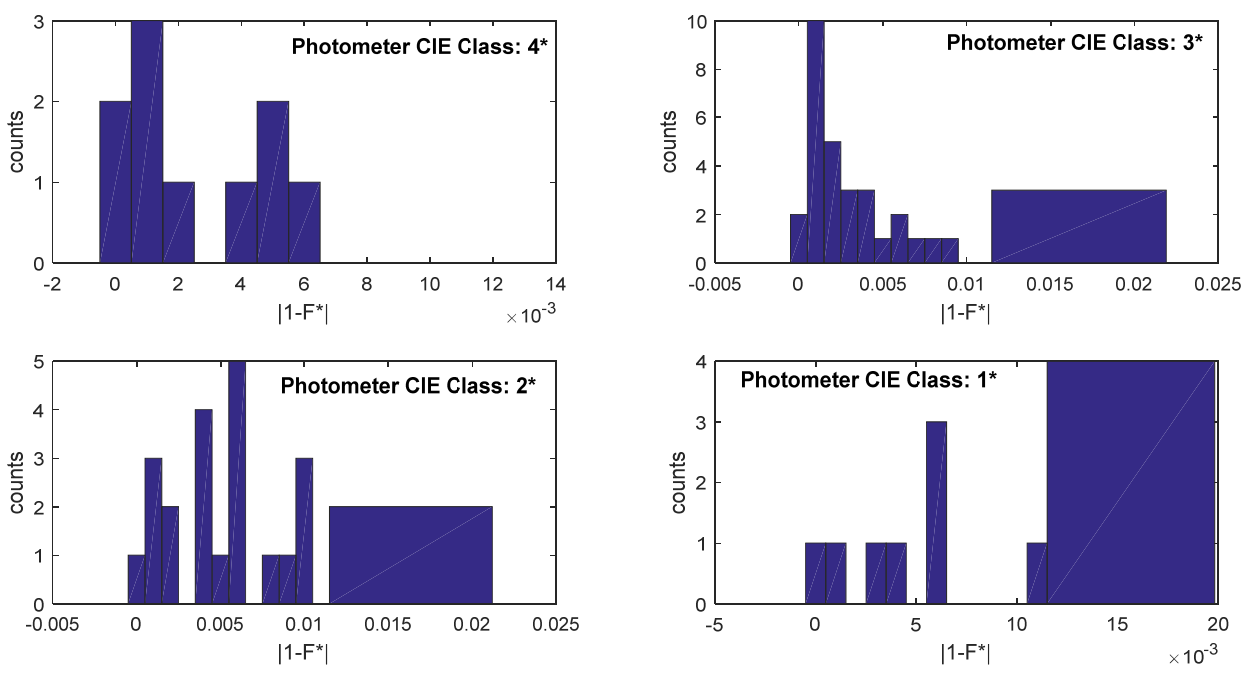

Figure 2 - Examples of distribution of values of $\left|1-F^{*}\right|$ when using photometers of the different CIE classes and a SPD with $\delta s m=0,181$. This SPD is shown in Figure 6. 

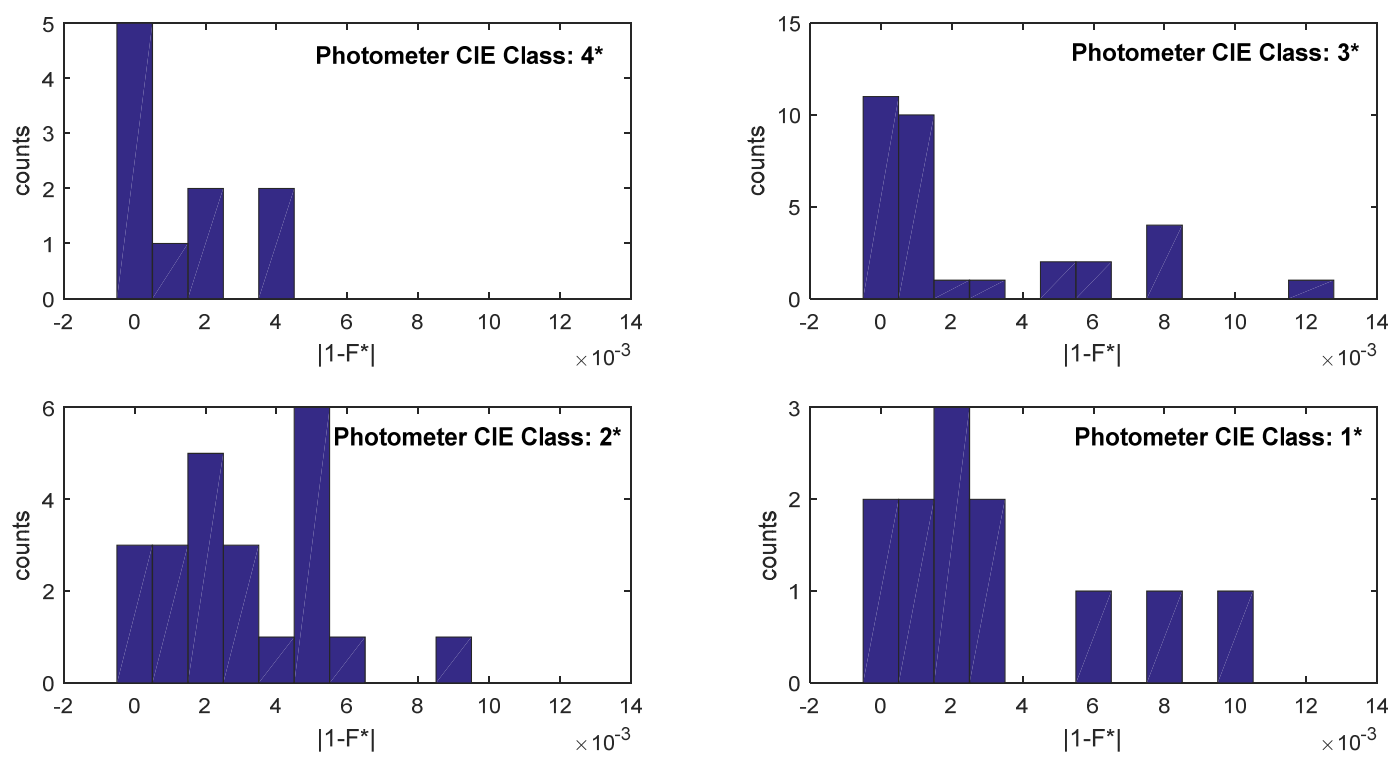

Figure 3 - Examples of distribution of values of $\left|1-F^{*}\right|$ when using photometers of the different $C I E$ classes and a SPD with $\delta s M=0,104$. This SPD is shown in Figure 6.
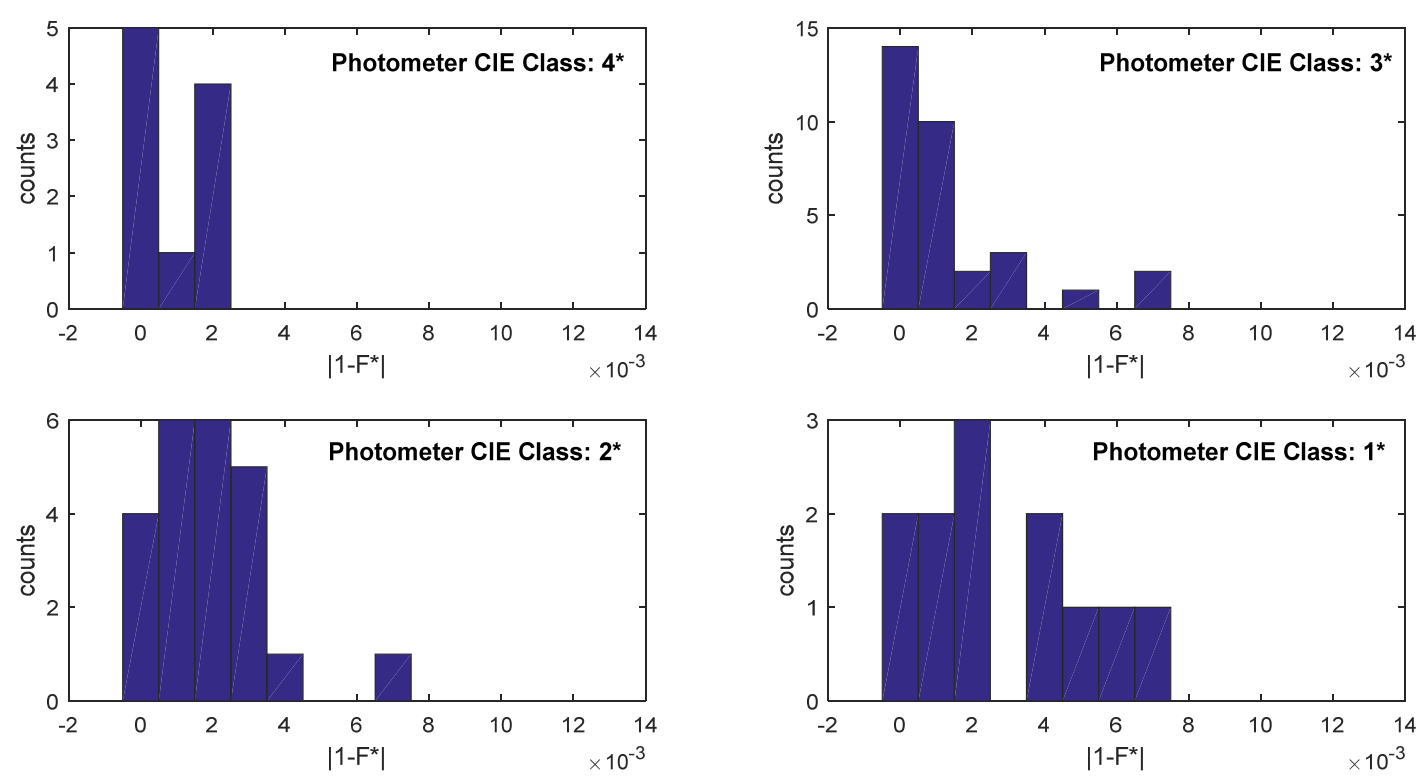

Figure 4 - Examples of distribution of values of $\left|1-F^{*}\right|$ when using photometers of the different $\mathrm{CIE}$ classes and a SPD with $\delta s_{M}=0,067$. This SPD is shown in Figure 6. 

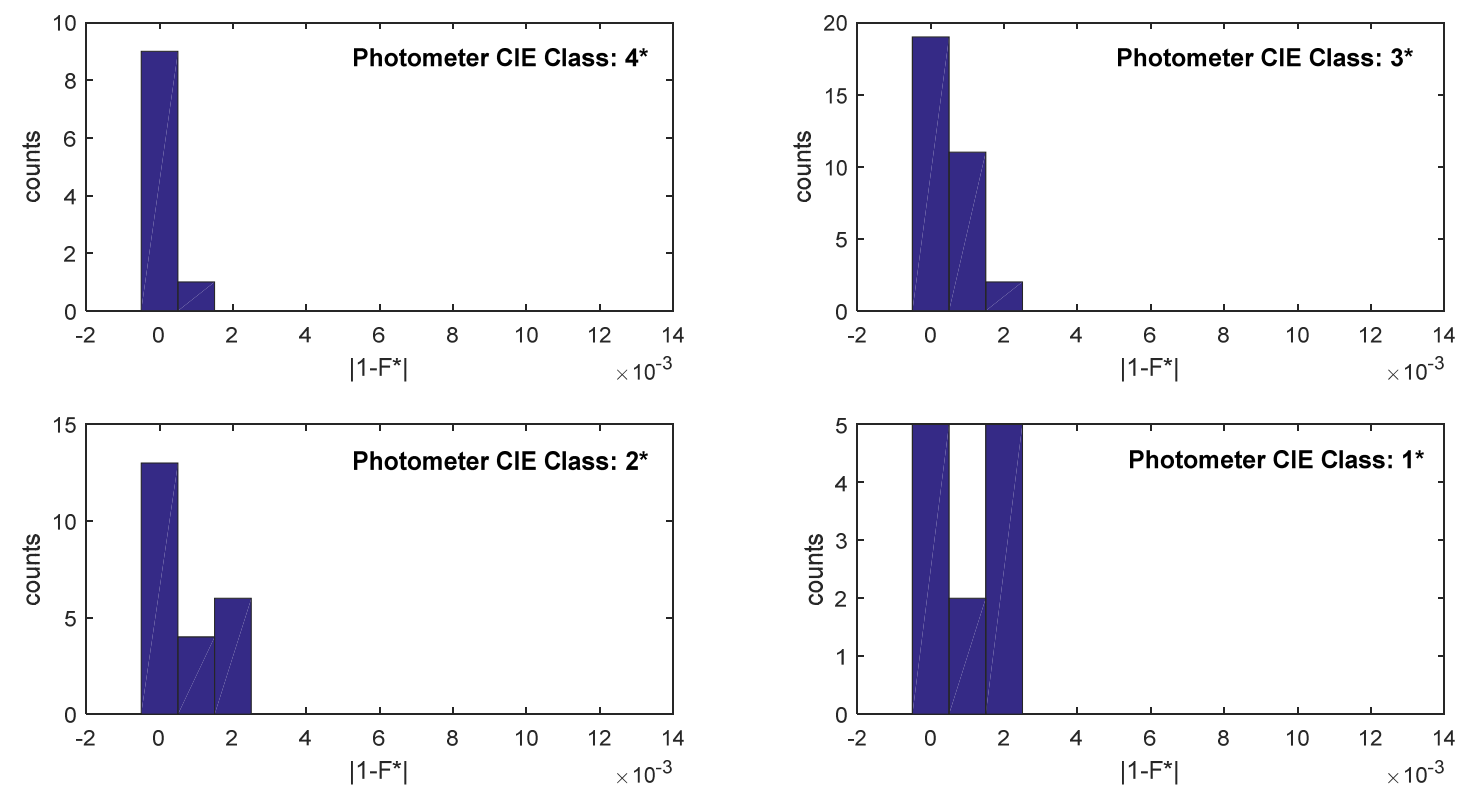

Figure 5 - Examples of distribution of values of $\left|1-F^{*}\right|$ when using photometers of the different $\mathrm{CIE}$ classes and a SPD with $\delta s_{M}=0,026$. This SPD is shown in Figure 6.

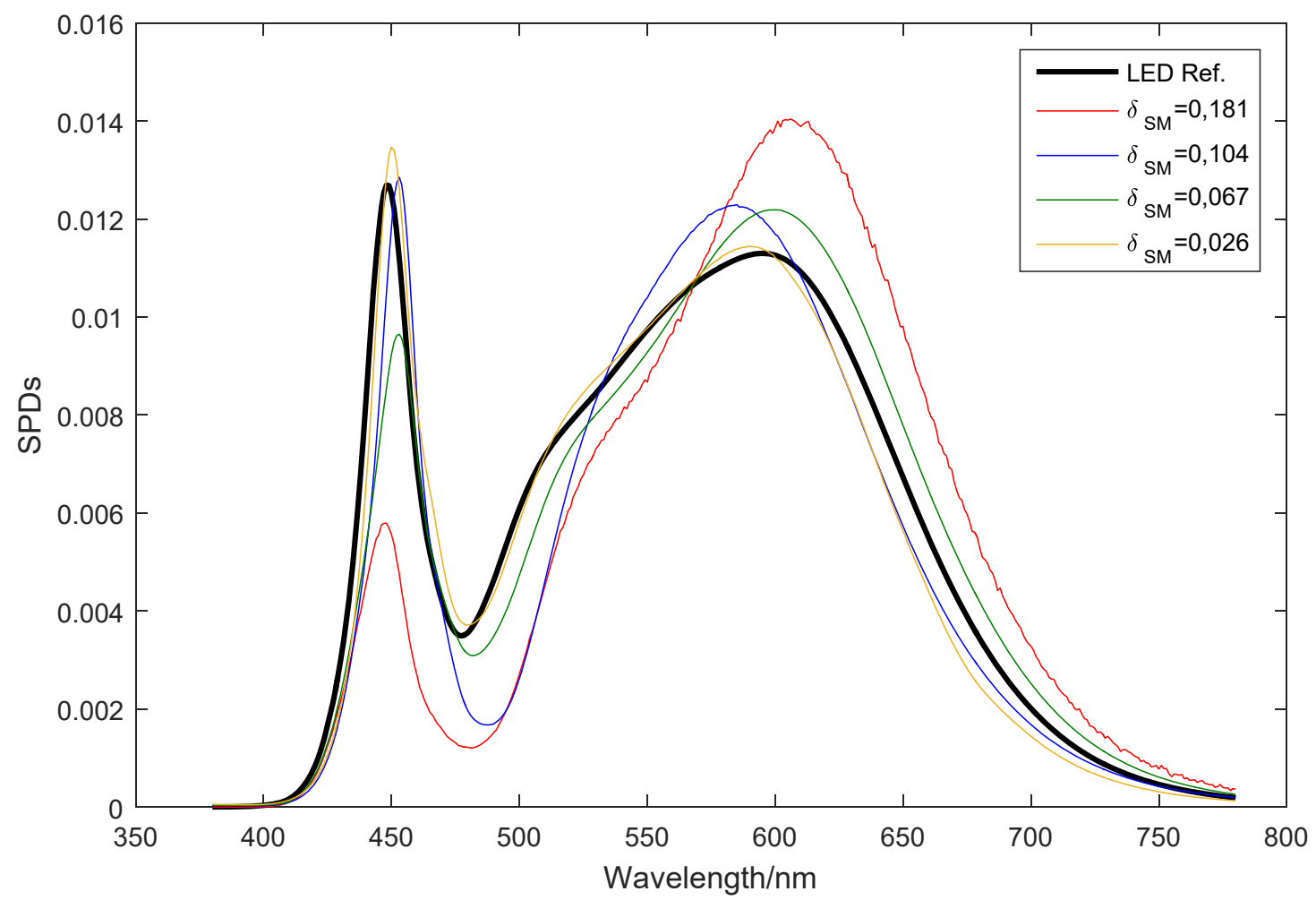

Figure 6 - Some SPDs of LEDs with different SPD spectral mismatch index $\delta$ sm. The LED reference spectrum is also shown for comparison.

Data and linear regressions for estimating $f_{50}$ and $f_{95}$ for different quality photometers are shown in Figure 7. Results are given in Table 1. 

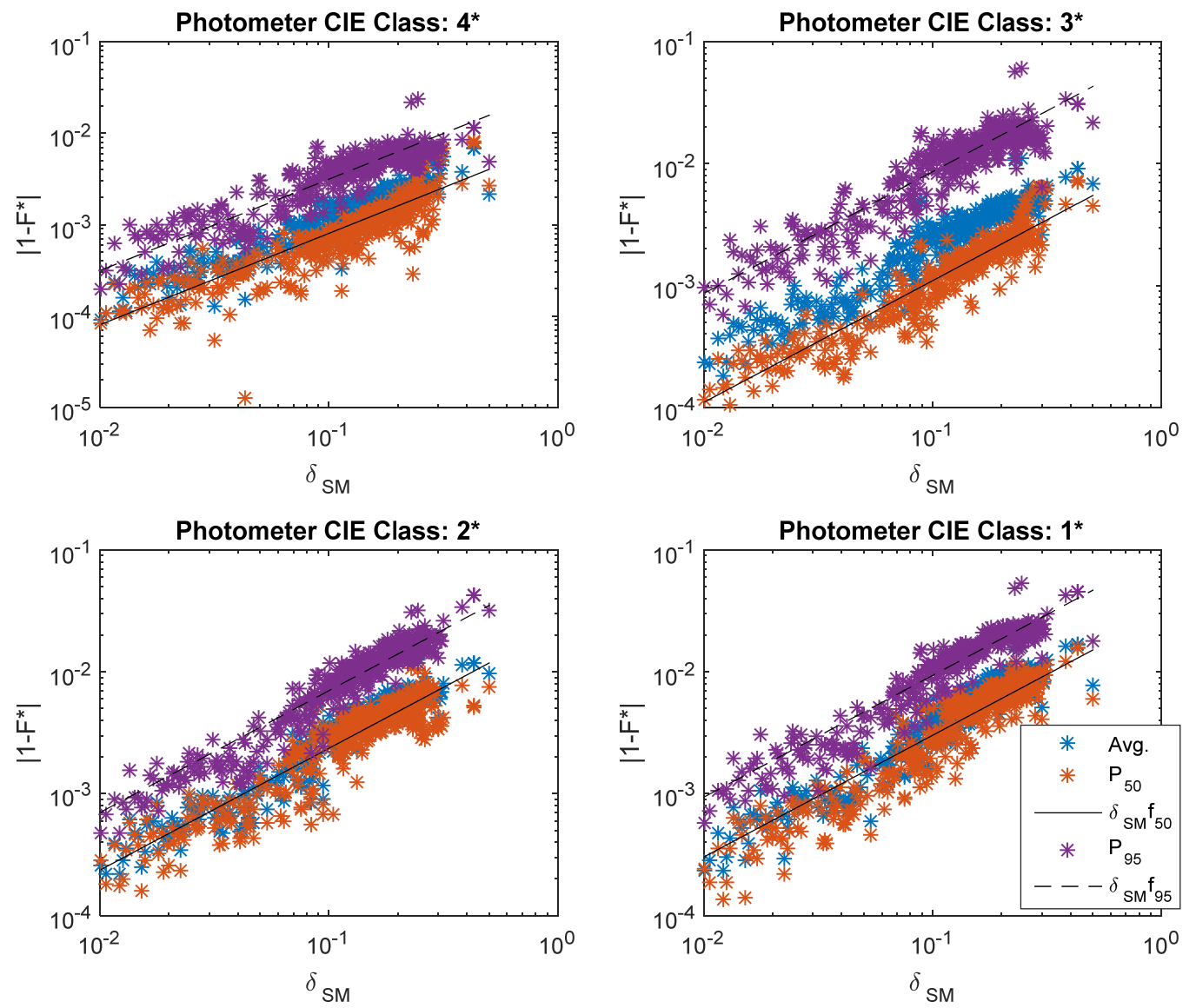

Figure 7 - Data and linear regressions for estimating $f_{50}$ and $f_{95}$ for different quality photometers.

Table 1 - Results of $f_{50}$ and $f_{95}$ for different quality photometers.

\begin{tabular}{ccc}
\hline $\begin{array}{c}\text { Photometer class } \\
\text { [CIE 2019] }\end{array}$ & $f_{50}($ BL40) & $f_{95}(\mathrm{BL40})$ \\
\hline $4^{*}$ & 0,015 & 0,05 \\
\hline $3^{*}$ & 0,017 & 0,13 \\
\hline $2^{*}$ & 0,040 & 0,13 \\
\hline $1^{*}$ & 0,050 & 0,13 \\
\hline
\end{tabular}

In Figure 7 , there is a clear dispersion in the data. Table 1 provides a practical guide to interpret the SPD spectral mismatch index $(\delta \mathrm{SM})$. It shows the values of $f_{50}$ and $f_{95}$ with a confidence of $95 \%$. It is observed that $f_{95}=0,13$ is the same for all classes but for class $4^{*}$. So, according to this Table, it can be said, for instance:

"If I am going to use only $4^{*}$ photometers, and the $\delta \mathrm{sm}$ of my calibration source is $0,05, I$ expect that the correction $\left|1-F^{*}\right|$ should be lower than $0,00075\left(f_{50} \times \delta \mathrm{SM}=0,015 \times 0,05\right)$ in $50 \%$ of the cases and lower than $0,0025\left(f_{95} \times \delta \mathrm{SM}=0,05 \times 0,05\right)$ in $95 \%$ of the cases."

or 
"If I am going to use only $\mathbf{3}^{*}$ photometers, and the $\delta \mathrm{sm}$ of my calibration source is $\mathbf{0 , 0 3}, I$ expect that the correction $\left|1-F^{*}\right|$ should be lower than $0,0012\left(f_{50} \times \delta \mathrm{sm}=0,04 \times 0,03\right)$ in $50 \%$ of the cases and lower than $0,0039\left(f_{95} \times \delta \mathrm{SM}=0,13 \times 0,03\right)$ in $95 \%$ of the cases."

\section{Conclusions}

A spectral mismatch quality index for SPDs has been proposed and validated using around 100000 values of experimental SMCFs (1298 SPDs combined with 77 spectral responsivities). It allows the match of spectral power distributions (SPDs) of real sources to the selected LED reference spectrum for photometric calibration to be evaluated. Given the linear correlation found between this index and the average value of $\left|1-F^{*}\right|$, it can be used to assign quality degrees to the real LED sources devoted to calibration purposes.

\section{Acknowledgment}

The work leading to this study is partly funded by the European Metrology Programme for Innovation and Research (EMPIR) Project 15SIB07 PhotoLED 'Future Photometry Based on Solid State Lighting Products'. The EMPIR initiative is cofunded by the European Union's Horizon 2020 research and innovation programme and the EMPIR Participating States.

\section{References}

KOKKA, A., et al. 2018. Development of white LED illuminants for colorimetry and recommendation of white LED reference spectrum for photometry. Metrologia, 55(4) 526534.

ISO/CIE 2014. ISO/CIE 19476:2014(E) Characterization of the Performance of Illuminance Meters and Luminance Meters.

CIE 2019. CIE TC 2-69, approval draft:2019 "CIE Classification Systems of Illuminance and Luminance Meters". 